\title{
Reciprocal relationship between depression and Internet gaming disorder in children: A 12-month follow-up of the iCURE study using cross-lagged path analysis
}

\author{
HYUNSUK JEONG ${ }^{1}$, HYEON WOO YIM ${ }^{1 *}$, SEUNG-YUP LEE ${ }^{2}$, HAE KOOK LEE ${ }^{2}$, MARC N. POTENZA ${ }^{3,4,5}$, \\ SUN-JIN JO ${ }^{1}$ and HYE JUNG SON ${ }^{1}$ \\ ${ }^{1}$ Department of Preventive Medicine, College of Medicine, The Catholic University of Korea, Seoul, South Korea \\ ${ }^{2}$ Department of Psychiatry, College of Medicine, The Catholic University of Korea, Seoul, South Korea \\ ${ }^{3}$ Departments of Psychiatry, Neuroscience and Child Study Center, Yale University, New Haven, CT, USA \\ ${ }^{4}$ Connecticut Council on Problem Gambling, Wethersfield, CT, USA \\ ${ }^{5}$ Connecticut Mental Health Center, New Haven, CT, USA
}

(Received: July 9, 2019; revised manuscript received: October 5, 2019; accepted: December 2, 2019)

\begin{abstract}
Objectives: Previous studies have reported an association between Internet gaming disorder (IGD) and depression, but the directionality of the relationship remains unclear. Therefore, we examined the reciprocal relationship between level of depressive symptoms and IGD among children in a longitudinal study. Methods: Research panels for this study consisted of 366 elementary-school students in the iCURE study. All participants were current Internet users, so they could be considered an at-risk population for IGD. Self-reported severity of IGD features and level of depression were assessed by the Internet Game Use-Elicited Symptom Screen and Children's Depression Inventory, respectively. Follow-up assessment was completed after 12 months. We fitted cross-lagged structural equation models to investigate the association between the two variables at two time points contemporaneously. Results: The cross-lagged analysis revealed that level of depression at baseline significantly predicted severity of IGD features at the 12-month follow-up $(\beta=0.15, p=.003)$. Severity of IGD features at baseline also significantly predicted level of depression at the 12-month follow-up $(\beta=0.11, p=.018)$, controlling for possible confounding factors. Conclusions: The cross-lagged path analysis indicates a reciprocal relationship between severity of IGD features and level of depressive symptoms. Understanding the reciprocal relationship between depressive symptoms and severity of IGD features can assist in interventions to prevent both conditions. These findings provide theoretical support for prevention and remediation plans for IGD and depressive symptoms among children.
\end{abstract}

Keywords: Internet gaming disorder, depressive symptoms, cross-lagged, cohort

\section{INTRODUCTION}

Children are developing in an era of digital technology and becoming familiar with computers, mobile devices, and the Internet at early ages. Gaming disorder is emerging as a major mental health problem in children and adolescents worldwide (Ioannidis et al., 2018), even though there remains debate whether game playing is beneficial or harmful to children and adolescents.

Half of all mental illness begins by the age of 14 years, and mood regulation problems sometimes begin around the age of 11 years, before puberty (Forbes \& Dahl, 2010; Guo et al., 2012). Mental health problems represent the largest burden of disease among young people. Previous studies have reported associations between Internet addiction and psychiatric symptoms, such as depression, anxiety, and loneliness, among adolescents. Among psychiatric symptom categories, depressive symptoms show the most powerful impact on the development of Internet addiction in children and adolescents (Erceg, Flander, \& Brezinšćak, 2018;
Niall McCrae, Gettings, \& Purssell, 2017; Piko, Milin, O'Connor, \& Sawyer, 2011).

Internet gaming disorder (IGD) and depression interact with each other and share neural mechanisms (Choi et al., 2017; Liu et al., 2018). Similar brain regions show abnormal functioning in both depression and IGD. The amygdala, prefrontal cortex, gyrus, and the connection between the frontoparietal lobe and the amygdala appear similarly disrupted in people with gaming problems and those with depression.

A systematic review showed that individuals with depressive symptoms are almost three times as likely to develop Internet addiction than those without depressive symptoms (Carli et al., 2013). However, 19 of 20 studies in

* Corresponding author: Hyeon Woo Yim, MD, PhD; Department of Preventive Medicine, College of Medicine, The Catholic University of Korea, 222 Banpodero, Seochogu, Seoul 06591, South Korea; Phone: +82 22258 7860; Fax: +82 2532 3820; E-mail: y1693@catholic.ac.kr

This is an open-access article distributed under the terms of the Creative Commons Attribution-NonCommercial 4.0 International License, which permits unrestricted use, distribution, and reproduction in any medium for non-commercial purposes, provided the original author and source are credited, a link to the CC License is provided, and changes - if any - are indicated. 
the review were cross-sectional studies that could not identify directionality of associations between depression and Internet addiction. Nonetheless, $75 \%$ of studies reported significant correlations between problematic Internet use and depression.

A limited number of longitudinal studies have assessed relationships between IGD and mental health outcomes among young people. A prospective cohort study in China found that college students who were initially free of mental health problems at baseline, as assessed by a self-report depression scale, were 2.5 times more likely to develop depression at the 9-month follow-up if they showed problematic use of the Internet at baseline (Lam, Peng, Mai, \& Jing, 2009). In a 2-year longitudinal study of children and adolescents, Gentile et al. (2011) found that problematic gaming statistically predicted future higher levels of depression, social phobia, and anxiety as measured by selfassessed mental health status (Gentile et al., 2011). Higher levels of depression have been associated with higher Internet addictive behaviors (Stavropoulos \& Adams, 2017).

Although previous studies may help identify factors related to the temporal order of relationships between depression and IGD, it is still unclear whether IGD is associated with the development of depression or whether the reverse relationship also holds. Therefore, we examined the stability and relationships between depressive symptoms and IGD over time to better understand how these two variables influence each other over time using a crosslagged path model. We evaluated the reciprocal relationship between depressive symptoms and severity of IGD features in prepubertal children to reduce the influence of mood changes during puberty.

\section{METHODS}

\section{Study population}

The study population was derived from the iCURE study, which has been described in detail elsewhere (Jeong et al., 2017). In brief, the iCURE study is an ongoing school-based longitudinal study to study the natural history of IGD among elementary students in the $3 \mathrm{rd}$ and 4 th grades and middleschool students in the 7th grade in Korea. All participants reported that they were current Internet users, so they were considered an at-risk population for IGD. The first followup assessment was completed 12 months after the baseline assessment. To reduce possible effects of pubertal mood changes on study results, research panels for this study consisted of students in the 3rd and 4th grades only who were part of the iCURE study. Of 399 elementary-school students who enrolled in the iCURE study at baseline, 366 $(91.5 \%)$ completed the 12-month follow-up assessment and were included in this study.

\section{Measurements}

At the baseline assessment, all participants completed the questionnaires in a class setting; a research assistant read the questions with a standard script to aid in comprehension. For the 12-month follow-up assessment, all students completed the questionnaires on their own, using a webbased self-administration method, with a supervising research assistant available to answer questions.

Severity of IGD features. Severity of IGD features was assessed by the Internet Game Use-Elicited Symptom Screen (IGUESS). This instrument was created based on the nine DSM-5 IGD criteria, with each item rated on a 4-point scale ( $1=$ strongly disagree, 2 =somewhat disagree, 3 = somewhat agree, $4=$ strongly agree). A higher score indicates greater severity of IGD features. This scale is reliable, with a Cronbach's $\alpha$ of .85 in this study. Severity of IGD was considered to have a continuous severity dimension, where higher scores on the IGUESS indicated greater severity for analysis with the cross-lagged path model. The best cut-off score was 10 to be considered at elevated risk of IGD (Jo et al., 2017). We used this threshold score for dichotomous analyses.

Level of depressive symptoms. Level of depression was assessed by the Children's Depression Inventory (CDI). The CDI has 27 items quantifying symptoms such as depressed mood, hedonic capacity, vegetative functions, self-evaluation, and interpersonal behaviors. Each item consists of three statements graded to increase severity from 0 to 2 ; children select the one that characterizes their symptoms best during the past 2 weeks. The item scores are combined into a total depression score, which ranges from 0 to 54 . We used the Korean version of the CDI, which has good reliability and validity for the assessment of depressive symptoms (Cho \& Choi, 1989). Level of depressive symptoms was considered to have a continuous severity dimension, where higher scores on the CDI indicated greater severity of depressive symptoms for analysis with the cross-lagged path model. A total score of 22 or more was considered to indicate depressive symptoms in the dichotomous analysis. Both severity of IGD features and level of depression were assessed at baseline and at the 12-month follow-up using interviewer-guided, self-report assessments.

Potential confounders. General characteristics, including age, sex, family type, and average daily time spent playing Internet games, were obtained from the baseline data acquired by the interviewer-guided children's self-report. For family type, an intact family was defined as children living with both parents; those defined as non-intact included children living with only a mother or father or with neither parent because of divorce, death, or separation of their parents. Puberty was determined according to the participants' responses to two questions: either "Have you started your period?" for girls or "Have you started growth of your underarm hair?" for boys. If participants responded "yes," we considered them to have entered puberty. Both academic performance of their children and socioeconomic status (SES) were obtained from the parents' self-report assessment.

\section{Statistical analyses}

Descriptive statistics and interrelationships between study variables were performed with SAS 9.4 (SAS Institute Inc., Cary, NC, USA). Cross-lagged panel modeling was conducted using structural equation modeling (SEM) with the aid of the Analysis of Moment Structures statistical 
package, version 23.0. (IBM Inc., Chicago, IL, USA). The descriptive data are summarized with numbers and percentages for categorical variables, or mean $\pm \mathrm{SD}$ or median (range) for continuous variables. The longitudinal association between severity of IGD features and level of depression was assessed with cross-lagged panel models. Before conducting the analysis, both level of depression and severity of IGD features were log-transformed to approximate normality.

Cross-lagged panel models allow associations between two or more repeatedly measured variables to be investigated contemporaneously. Therefore, the cross-lagged correlations indicate the impact of a variable at a given point in time on the values of another variable later in time, controlling for cross-sectional correlations and autocorrelations.

As illustrated in Figure 1A, the first cross-lagged coefficient $\beta C L$ (a) represents the association between level of depression measured at baseline and severity of IGD features measured at the 12-month follow-up. The second cross-lagged coefficient $\beta C L$ (b) represents the association between severity of IGD features measured at baseline and level of depression measured at the 12-month follow-up. The cross-sectional association between severity of IGD features and level of depression is represented as $\beta C L$ baseline. Autoregressive coefficients $\beta$ AR-depression and $\beta A R-I G D$, representing the stability of depression and severity of IGD features from baseline to 12-month follow-up, respectively, are presented. The model was adjusted for potential confounding factors, such as age, sex, family type, academic achievement, and SES.

To test the mediation effect, 2,000 bootstrapped resamples and a $95 \%$ confidence interval (CI) were applied to construct the indirect path. Bias-corrected CIs that did not include 0 were considered significant for the indirect effect. Effect sizes interpreted as small (0.01), medium (0.09), and large $(0.25)$ based on the previous recommendation (Preacher \& Kelley, 2011).
Model fit was assessed using multiple fit indices including absolute fit indices, incremental fit indices, and parsimony fit indices. Incremental fit indices were assessed using the $\chi^{2}$ over degrees of freedom $\left(\chi^{2} / d f\right)$ ratio, goodness of fit index (GFI), comparative fit index (CFI), and root mean square error of approximation (RMSEA). Incremental fit indices were assessed using the Tucker-Lewis index (TLI), normed fit index, relative fit index (RFI), and comparative fit index (CFI). An adjusted GFI (AGFI) was used for parsimony fit indices. The SEM literature suggests that model fit is good when $\chi^{2} / d f \leq 3$; CFI $\geq 0.95$, TLI $\geq 0.95$, GFI $\geq 0.95$, $\mathrm{NFI} \geq 0.95, \mathrm{RFI} \geq 0.95, \mathrm{AGFI} \geq 0.95$, and RMSEA $\leq 0.06$ (Kline, 2011).

For an additional analysis, elevated risk of IGD was defined as having a total score of 10 or higher on the IGUESS scale, and high level of depressive symptoms was defined as having a total score on the CDI of 22 or higher. We used a log-binomial model run with PROC GENMOD to estimate relative risk (RR) for the association between a high level of depressive symptoms and incident elevated risk of IGD during the 12-month follow-up period among children with lower risk of IGD ( $<10$ IGURSS scores) at baseline. The incidence rate of a high level of depressive symptoms at the 12-month follow-up was calculated among children with no depressive symptoms at baseline. We calculated crude and adjusted RRs while controlling for potential confounding factors.

\section{Ethics}

To enroll in the iCURE study, written informed consent was acquired from all participants and their parents or legal guardians after explanation of the nature of the principles of research, including confidentiality and the freedom of choice to participate in accordance with the Declaration of Helsinki of 1975 (World Medical Association, 2013). This study was fully reviewed and approved by the Institutional Review Board of The Catholic University of Korea
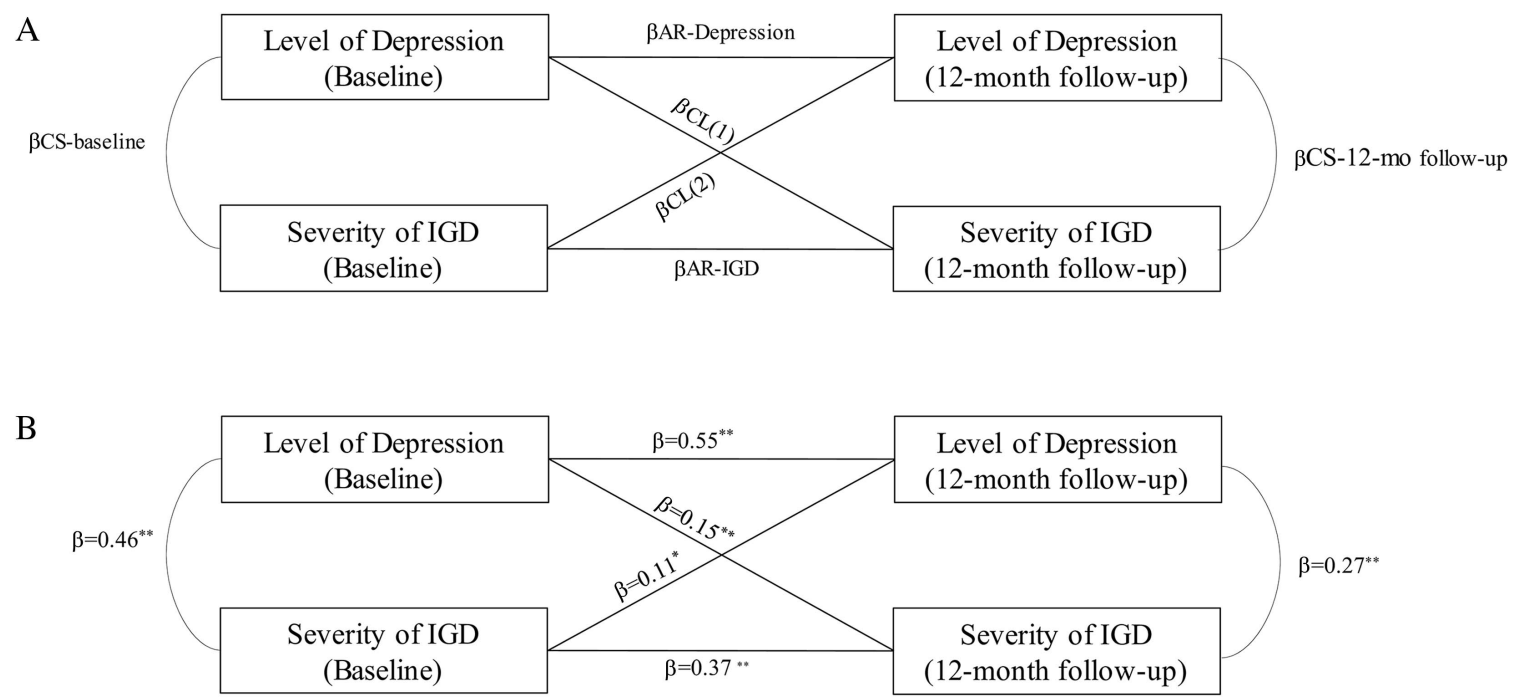

Figure 1. (A) The general modeling used for cross-lagged panel models. (B) The cross-lagged panel model analyzing the longitudinal association between IGD and depression. Numeric values are standardized structural regression coefficients. AR: autoregressive;

CL: cross-lagged; CS: cross-sectional. ${ }^{*} p<.05 .{ }^{* *} p<.01$. 
(MC19ENSI0071). The iCURE data management board released de-identified data for data analysis.

\section{RESULTS}

Demographic and clinical characteristics of the 366 participants are summarized in Table 1. The median age of participants was 10 years (range: 9-12 years). Of the 366 participants, $188(51.4 \%)$ were boys. Most participants $(n=337 ; 92.1 \%)$ were from intact families, $68 \%$ of participants had good academic performance, and $71 \%$ reported that their SES was low to moderate.

Correlations between major variables of interest are reported in Table 2. Cross-sectionally, level of depression at baseline was positively correlated with severity of IGD at both baseline and 12-month follow-up. Longitudinally, level of depression (baseline) was positively correlated with severity of IGD (12-month follow-up), and severity of IGD (baseline) was positively correlated with level of depression (12-month follow-up).

Figure 1 shows the theorized model (A) and analyzed model (B) with standardized path loadings (standardized beta, $\beta)$. Regarding the autocorrelated paths, level of depression at baseline statistically predicted severity of IGD features at the 12-month follow-up $(\beta=0.55$, $p<.001)$. In addition, severity of IGD features at baseline statistically predicted level of depression at the 12-month follow-up $(\beta=0.37, p<.001)$. The results showed that both level of depressive symptoms and severity of IGD features were significantly correlated between baseline and the 12-month follow-up. Similarly, severity of IGD features was correlated across the two time points.

Regarding the cross-sectional correlation path, the level of depressive symptoms and severity of IGD features were positively correlated at each time point $(\beta=0.46, p<0.001$ at baseline and $\beta=0.27, p<.001$ at 12 -month follow-up). The results showed a positive correlation between level of depressive symptoms and severity of IGD features at each time point.

The cross-lagged analyses revealed that level of depression at baseline statistically predicted severity of IGD features at the 12-month follow-up $(\beta=0.15, p=.003)$. Severity of IGD features at baseline also statistically predicted level of depression at the 12-month follow-up

Table 1. General and clinical characteristics of 366 elementary-school students in the iCURE study

\begin{tabular}{lccc}
\hline Variables & $N(\%)$ & Median (range) & Cronbach's $\alpha$ \\
\hline Sex & $188(51.4)$ & & \\
Boys & $178(48.6)$ & & \\
Girls & & $10(9-12)$ & \\
Age & $337(92.1)$ & & \\
Family structure & $29(7.9)$ & & \\
Intact family & & & \\
Non-intact family & $263(71.9)$ & & .78 \\
Socioeconomic status & $103(28.1)$ & & .88 \\
Low and middle & & & .89 \\
High & $249(68.0)$ & & \\
Academic achievement & $117(32.0)$ & & .86 \\
Good & & $2(0-22)$ & .89 \\
Bad & & $6(0-46)$ & .94 \\
Baseline assessments & & $26(20-58)$ & \\
Internet gaming disorder & & & \\
Depression & & $5(0-23)$ & \\
Anxiety & & $24(20-58)$ & \\
12-Month follow-up assessments & & & \\
Internet gaming disorder & & & \\
Depression & & & \\
Anxiety & & & \\
\hline
\end{tabular}

Table 2. Correlation matrix, mean, and standard deviation $(S D)$ for main variables

\begin{tabular}{|c|c|c|c|c|c|c|}
\hline Variables & 1 & 2 & 3 & 4 & Mean & $S D$ \\
\hline 1. Level of depression (baseline) & 1 & & & & 7.4 & 6.5 \\
\hline 2. Severity of IGD (baseline) & $.443^{*}$ & 1 & & & 2.6 & 3.2 \\
\hline 3. Level of depression (12-month follow-up) & $.596^{*}$ & $.339 *$ & 1 & & 6.7 & 6.6 \\
\hline 4. Severity of IGD (12-month follow-up) & $.359^{*}$ & $.453^{*}$ & $.447^{*}$ & 1 & 2.9 & 3.6 \\
\hline
\end{tabular}

Note. IGD: Internet gaming disorder.

${ }^{*} p<.001$. 
$(\beta=0.11, p=.018)$, after controlling for possible confounding factors. The cross-lagged path analysis indicated a reciprocal relationship between severity of IGD features and level of depressive symptoms.

Our overall model demonstrated good fit based on fit indices. The ratio of the $\chi^{2}$ to the degrees of freedom was 1.336 , indicating a good model fit. The RMSEA was 0.03 , GFI was 0.997, TLI was 0.976, CFI was 0.997, and AGFI was 0.964 , also indicating good fit. When taken together, the fit statistics suggest that this was sufficient to produce a valid model based on a strong a priori theoretical framework and acceptable reliabilities.

Of 366 participants, 351 did not report being at elevated risk of IGD at baseline. Of these 351 participants, 15 (4.3\%) were classified as being at elevated risk of IGD at the 12-month follow-up. After adjusting for potential confounding factors, participants with depressive symptoms at baseline had an 3.7-fold greater RR of IGD at 12 months than participants without depressive symptoms at baseline $(\mathrm{RR}=3.7,95 \% \mathrm{CI}=1.1-13.2)$.

Of 366 participants, 353 did not report a high level of depressive symptoms at baseline. Of these 353 participants, $8(2.3 \%)$ were classified as having a high level of depressive symptoms at the 12-month follow-up. After adjusting for potential confounding factors, participants who were at elevated risk of IGD at baseline had a 3.6-fold increased risk of depression at the 12-month follow-up compared to participants who were not at elevated risk of IGD at baseline, however it was not statistically significant ( $R R=3.6,95 \% \mathrm{CI}=0.5-29.0$; Table 3).

\section{DISCUSSION}

We found a significant positive correlation between level of depressive symptoms and severity of IGD features at both baseline and the 12-month follow-up in children. These results suggest that depressive symptoms constitute a potential risk factor for elevated IGD severity, and severity of IGD features may constitute a potential risk factor for depressive symptoms one year later.

A cross-lagged path analysis allows for multiple relationships to be analyzed simultaneously, producing more complex statistical models than could be obtained from running several separate linear regressions. The relative strengths of longitudinal relationships can be determined through comparison of standardized correlation coefficients. Both severity of IGD features and level of depressive symptoms showed significant cross-sectional, auto-correlation, and cross-lagged correlation coefficients.

Cross-sectional correlations revealed a positive association between the level of depressive symptoms and severity of IGD features at each time point. Similarly, autocorrelation revealed that both level of depressive symptoms and severity of IGD features were significantly correlated with stability across the two time points. The cross-lagged path analysis indicated a reciprocal causality between risk of IGD and level of depressive symptoms. These crosssectional and longitudinal associations persisted after controlling for potential confounders. The strength of the relationship was stronger between baseline level of depression and 12-month severity of IGD features $(\beta=0.15$, $p=.003)$ than between baseline severity of IGD features and 12-month level of depression $(\beta=0.11, p=.018)$, which are suggested to be a medium effect size. This finding suggests that depression is a strongest contributor to severity of IGD features than vice versa, and that there is a reciprocal relationship over time.

Associations between IGD and depression have sometimes been explained by a mood enhancement hypothesis, which suggests that individuals with negative emotions are most likely to seek recreational activities to escape from dysphoric states. Previous studies have been consistent with a mood enhancement hypothesis in that a significant, positive relationship between depression and IGD has been observed (Ostovar et al., 2019; Seyrek, Cop, Sinir, Ugurlu, \& Senel, 2017; Yen, Chou, Liu, Yang, \& Hu, 2014; Younes et al., 2016). Attempts to escape depression and real-world concerns through online interactions may result in a vicious cycle that exacerbates depression.

According to the social displacement hypothesis, the more time a person spends doing one thing, the less time that may be spent doing another. Children who spend excessive time on Internet gaming typically spend less time interacting with other people (Caplan, 2003). An assumption of the social displacement effect is that time spent on gaming will displace other activities, such as social interaction, that are essential to psychosocial development in children (Zamani, Kheradmand, Cheshmi, Abedi, \& Hedayati, 2010). A lack of social interaction may lead to negative emotions. Gentile et al. (2011) reported elevated depressive symptoms after video-gaming problems started,

Table 3. Incidence of both IGD and depression among children at 12-month follow-up

\begin{tabular}{lcccccc}
\hline & Yes & No & IR & RR & aRR $^{\mathrm{a}}$ \\
\hline 12-month IGD $^{\mathrm{b}}$ & & & & & & \\
Baseline depression & Yes & 2 & 8 & 20 & $5.2(1.4-20.2)$ & $3.7(1.1-13.2)$ \\
& No & 13 & 328 & 3.8 & & \\
12-month depression & & & & & & \\
Baseline IGD $^{\mathrm{c}}$ & Yes & 1 & 11 & 8.3 & $4.1(0.5-30.4)$ & $3.6(0.5-29.0)$ \\
& No & 7 & 334 & 2.1 & & \\
\hline
\end{tabular}

Note. IR: incidence rate; RR: relative risk; aRR: adjusted relative risk; IGD: Internet gaming disorder.

${ }^{a}$ Adjusted by sex, family type, academic achievement, and socioeconomic status.

${ }^{\mathrm{b}}$ Incidence rate of IGD at the 12-month follow-up among children without IGD at baseline $(n=351)$.

${ }^{\mathrm{c}}$ Incidence rate of depression at the 12-month follow-up among children without depression at baseline $(n=353)$. 
and these symptoms persisted (Gentile et al., 2011). If the social displacement hypothesis is correct, then IGD may lead to depression (Amorosi, Ruggieri, Franchi, \& Masci, 2012; Dalbudak et al., 2013).

Depressive symptoms in adolescents tend to occur before puberty. In terms of genetic vulnerability for a major depressive disorder, the experience of stressful life events or the presence of psychiatric disorders during childhood have been linked to the onset of depression (Piko et al., 2011; Shapero et al., 2014). Since prepubertal depression has been linked to the development of antisocial disorder and addiction (Ryan, 2003), it is likely that prevention efforts for Internet addiction should be implemented at young ages to minimize exacerbating effects on depression. Thus, more attention should be paid to depression and its potential impacts on IGD development in children.

Children with depressive symptoms at baseline showed a 3.7-fold increased risk of developing IGD symptoms at the 12-month follow-up compared to children without depressive symptoms at baseline, after adjusting for potential confounding factors. Since the $95 \% \mathrm{CI}$ is as wide as $1.1-13.2$, there might be limitations to ensure the accuracy of the estimates, so these results should be interpreted with caution. In addition, children with IGD symptoms at baseline could be at increased risk of developing depressive symptoms at the 12-month follow-up compared to children without IGD symptoms at baseline; however, the results was not statistically significant.

Girls attain puberty about 12 years earlier than boys. The average age for girls to begin puberty is 12.7 years in representative nationwide samples (Lee, Kim, Oh, Lee, \& Park, 2016). From this perspective, most participants in this study would not yet have undergone puberty. A total of 8 $(2.2 \%)$ children were found to have reached puberty (3 at baseline; 5 at the 12-month follow-up). Because of the small number of children having entered puberty, the results of this study were likely not influenced by puberty-related changes.

The attrition rate at the 12-month follow-up was $9.1 \%$ (33 children). All the attrition occurred because the students had transferred to another school. There were no significant differences in baseline characteristics, including, sex, age, family type, academic performance, SES, Internet activity, or severity of IGD features, between the participants who did and did not complete the study.

The factors related to depression may differ among countries. Depression is a multifactorial condition, which shows considerable variation among different populations and is associated with a multitude of genetic and socioenvironmental factors, with several subtypes with differing etiologies. Korea was the first country to allocate a national budget to address the problems of Internet and gaming addiction (Koh, 2015). Psychosocial, environmental, and cultural differences may affect the relationships between depressive symptoms and severity of IGD features, although a fundamental association between depression and IGD may be expected to be observed across jurisdictions and cultures. Thus, the results of this study may be relevant to children in other countries, although caution is advised when generalizing the findings. Because the respondents were sampled among adolescents who were attending schools and excluded children not in school. The participating schools as well as the children and parents were voluntarily involved; therefore, these schools had interest in preventing IGD compared with nonparticipating schools. The possibility of selection bias and underestimation of IGD prevalence cannot be ruled out.

\section{CONCLUSIONS}

Childhood is a risk period for development of both depression and IGD. These two disorders frequently co-occur in childhood and are associated with significant functional impairment in later life. Given the ongoing development of mental characteristics throughout adolescence and early adult life, a better understanding of the directionality of the onset and course of these disorders during childhood will be helpful in developing more effective preventive and treatment strategies.

Cross-lagged path analysis indicated bidirectional relationships between severity of IGD features and level of depression. A higher level of depressive symptoms at baseline predicted higher severity of IGD features after 12 months. Moreover, baseline severity of IGD features was significantly related to a higher level of depressive symptoms after 12 months in children. Understanding the reciprocal relationships between depressive symptoms and severity of IGD features can assist in interventions to prevent both conditions. These findings provide theoretical support for prevention and remediation plans for IGD and depressive symptoms among children.

Funding sources: This study was supported by a grant from the Korean Mental Health Technology R\&D Project, Ministry of Health and Welfare, Republic of Korea (HL19C0012). MNP received support from the National Center for Responsible Gaming, Connecticut Council on Problem Gambling and Connecticut Department of Mental Health and Addiction Services.

Authors' contribution: HJ conducted the analyses and led the writing of the manuscript. HWY guided and supervised the writing of the manuscript. HJ and HWY developed and proposed the basic idea of the study. S-YL, HL, and MNP reviewed scientific content and edited the manuscript. HWY, HJ, S-YJ, and HS conducted the study. All authors contributed editorial comments on the manuscript.

Conflict of interest: The authors declare no conflict of interest with respect to the content of the manuscript. Dr. MNP reports the following disclosures. He has consulted for and advised Game Day Data, the Addiction Policy Forum, RiverMend Health, Lakelight Therapeutics/Opiant and Jazz Pharmaceuticals; has received research support from the Mohegan Sun Casino and the National Center for Responsible Gaming; has participated in surveys, mailings, or telephone consultations related to drug addiction, impulse control disorders, or other health topics; and has consulted for law offices and gambling entities on issues related to impulse control or addictive disorders. 
Data availability: The data sets generated during and/or analyzed during this study are available from the corresponding author.

\section{REFERENCES}

Amorosi, M., Ruggieri, F., Franchi, G., \& Masci, I. (2012). Depression, pathological dependence, and risky behaviour in adolescence. Psychiatria Danubina, 24(Suppl. 1), S77-S81.

Caplan, S. E. (2003). Preference for online social interaction a theory of problematic Internet use and psychosocial well-being. Communication Research, 30(6), 625-648. doi:10.1177/ 0093650203257842

Carli, V., Durkee, T., Wasserman, D., Hadlaczky, G., Despalins, R., \& Kramarz, E. (2013). The association between pathological internet use and comorbid psychopathology: A systematic review. Psychopathology, 46(1), 1-13. doi:10.1159/000337971

Cho, S., \& Choi, J. (1989). Development of State-Trait Anxiety Scale for Korean children. Medicine Journal of Seoul National University, 14(3), 150-157.

Choi, J., Cho, H., Kim, J. Y., Jung, D. J., Ahn, K. J., Kang, H. B., Choi, J. S., Chun, J. W., \& Kim, D. J. (2017). Structural alterations in the prefrontal cortex mediate the relationship between Internet gaming disorder and depressed mood. Scientific Reports, 7(1), 1245. doi:10.1038/s41598-017-01275-5

Dalbudak, E., Evren, C., Aldemir, S., Coskun, K. S., Ugurlu, H., \& Yildirim, F. G. (2013). Relationship of Internet addiction severity with depression, anxiety, and alexithymia, temperament and character in university students. Cyberpsychology, Behavior, and Social Networking, 16(4), 272-278. doi:10.1089/cyber.2012.0390

Erceg, T., Flander, G., \& Brezinšćak, T. (2018). The relationship between compulsive Internet use and symptoms of depression and anxiety in adolescence. Alcoholism and Psychiatry Research, 54(2), 101-112. doi:10.20471/dec.2018.54.02.02

Forbes, E. E., \& Dahl, R. E. (2010). Pubertal development and behavior: Hormonal activation of social and motivational tendencies. Brain and Cognition, 72(1), 66-72. doi:10.1016/j.bandc. 2009.10.007

Gentile, D. A., Choo, H., Liau, A., Sim, T., Li, D., Fung, D., \& Khoo, A. (2011). Pathological video game use among youths: A two-year longitudinal study. Pediatrics, 127(2), e319-e329. doi:10.1542/peds.2010-1353

Guo, J., Chen, L., Wang, X., Liu, Y., Chui, C. H., He, H., Qu, Z., \& Tian, D. (2012). The relationship between Internet addiction and depression among migrant children and left-behind children in China. Cyberpsychology, Behavior, and Social Networking, 15(11), 585-590. doi:10.1089/cyber.2012.0261

Ioannidis, K., Treder, M. S., Chamberlain, S. R., Kiraly, F., Redden, S. A., Stein, D. J., Lochner, C., \& Grant, J. E. (2018). Problematic Internet use as an age-related multifaceted problem: Evidence from a two-site survey. Addictive Behaviors, 81, 157-166. doi:10.1016/j.addbeh.2018.02.017

Jeong, H., Yim, H. W., Jo, S. J., Lee, S. Y., Kim, E., Son, H. J., Han, H. H., Lee, H. K., Kweon, Y. S., Bhang, S. Y., Choi, J. S., Kim, B. N., Gentile, D. A., \& Potenza, M. N. (2017). Study protocol of the Internet user Cohort for Unbiased Recognition of gaming disorder in Early adolescence (iCURE), Korea, 2015-2019. BMJ Open, 7(10), e018350. doi:10.1136/ bmjopen-2017-018350
Jo, S. J., Yim, H. W., Lee, H. K., Lee, H. C., Choi, J. S., \& Baek, K. Y. (2017). The Internet Game Use-Elicited Symptom Screen proved to be a valid tool for adolescents aged 10-19 years. Acta Paediatrica, 107(3), 511-516. doi:10.1111/apa.14087

Kline, R. B. (2011). Principles and practice of structural equation modeling (3rd ed.). New York, NY/London, UK: The Guilford Press.

Koh, Y. (2015). The Korean national policy for Internet addiction. In C. Montag \& M. Reuter (Eds.), Internet addiction neuroscientific approaches and therapeutic interventions (pp. 219-234). London, UK: Springer.

Lam, L. T., Peng, Z. W., Mai, J. C., \& Jing, J. (2009). Factors associated with Internet addiction among adolescents. CyberPsychology \& Behavior, 12(5), 551-555. doi:10.1089/cpb. 2009.0036

Lee, M. H., Kim, S. H., Oh, M., Lee, K. W., \& Park, M. J. (2016). Age at menarche in Korean adolescents: Trends and influencing factors. Reproductive Health, 42(1), 121-126. doi:10.1530/ jrf.0.0420121

Liu, L., Yao, Y. W., Li, C. R., Zhang, J. T., Xia, C. C., Lan, J., Ma, S. S., Zhou, N., \& Fang, X. Y. (2018). The comorbidity between Internet gaming disorder and depression: Interrelationship and neural mechanisms. Front Psychiatry, 9, 154. doi:10.3389/fpsyt.2018.00154

Niall McCrae, N., Gettings, S., \& Purssell, E. (2017). Social media and depressive symptoms in childhood and adolescence: A systematic review. Adolescent Research Review, 2(4), 315-330. doi:10.1007/s40894-017-0053-4

Ostovar, S., Allahyar, N., Aminpoor, H., Moafian, F., Nor, M., \& Griffiths, M. D. (2019). Internet addiction and its psychosocial risks (depression, anxiety, stress and loneliness) among Iranian adolescents and young adults: A structural equation model in a cross-sectional study. International Journal of Mental Health and Addiction, 14(3), 257-267. doi:10.1007/s11469-0159628-0

Piko, B. F., Milin, R., O’Connor, R., \& Sawyer, M. (2011). Multidisciplinary approach to child and adolescent depression. Depression Research and Treatment, 2011, 1-3. doi:10.1155/ 2011/854594

Preacher, K. J., \& Kelley, K. (2011). Effect size measures for mediation models: Quantitative strategies for communicating indirect effects. Psychological Methods, 16(2), 93-115. doi:10.1037/a0022658

Ryan, N. D. (2003). Child and adolescent depression: Short-term treatment effectiveness and long-term opportunities. International Journal of Methods in Psychiatric Research, 12(1), 44-53. doi:10.1002/mpr.141

Seyrek, S., Cop, E., Sinir, H., Ugurlu, M., \& Senel, S. (2017). Factors associated with Internet addiction: Cross-sectional study of Turkish adolescents. Pediatrics International, 59(2), 218-222. doi:10.1111/ped.13117

Shapero, B. G., Black, S. K., Liu, R. T., Klugman, J., Bender, R. E., Abramson, L. Y., \& Alloy, L. B. (2014). Stressful life events and depression symptoms: The effect of childhood emotional abuse on stress reactivity. Journal of Clinical Psychology, 70(3), 209-223. doi:10.1002/jclp.22011

Stavropoulos, V., \& Adams, B. L. M. (2017). Internet gaming disorder symptoms in emergent adulthood: The interplay between anxiety and family cohesion. Journal of Behavioral Addictions, 6(2), 237-247. doi:10.1556/2006.6. 2017.026 
World Medical Association (2013). Declaration of Helsinki: Ethical principles for medical research involving human subjects. JAMA, 310(20), 2191-2194. doi:10.1001/jama.2013. 281053

Yen, C. F., Chou, W. J., Liu, T. L., Yang, P., \& Hu, H. F. (2014). The association of Internet addiction symptoms with anxiety, depression and self-esteem among adolescents with attentiondeficit/hyperactivity disorder. Comprehensive Psychiatry, 55(7), 1601-1608. doi:10.1016/j.comppsych.2014.05.025
Younes, F., Halawi, G., Jabbour, H., El Osta, N., Karam, L., Hajj, A., \& Rabbaa Khabbaz, L. (2016). Internet addiction and relationships with insomnia, anxiety, depression, stress and self-esteem in university students: A cross-sectional designed study. PLoS One, 11(9), e0161126. doi:10.1371/journal.pone.0161126

Zamani, E., Kheradmand, A., Cheshmi, M., Abedi, A., \& Hedayati, N. (2010). Comparing the social skills of students addicted to computer games with normal students. Addiction and Health, 2(3-4), 59-65. doi:10.1016/S0924-9338(12)74212-8 